\title{
Efficiency of GaAs-Based Pulsed Terahertz Emitters
}

\author{
A. Reklaitis* \\ Semiconductor Physics Institute \\ A. Goštauto 11, LT-01108, Vilnius, Lithuania

\begin{abstract}
Terahertz emission from the electron-hole plasma excited by a femtosecond optical pulse in GaAs-based emitters is studied by the Monte Carlo simulations. The $\mathrm{THz}$ energy radiated from the $n$ - and $p$-doped GaAs surface $\mathrm{THz}$ emitters, from the contactless $p-i-n$ emitter, and from the photoconductive emitter is evaluated. The obtained results show that the THz energy radiated by the photoconductive emitter exceeds the energy radiated by the surface and $p-i-n \mathrm{THz}$ emitters by more than one order of magnitude.
\end{abstract}

PACS numbers: 42.72.Ai, 73.20.Mf, 78.20.Bh, 78.47.-p

\section{Introduction}

Several emitter concepts for pulsed $\mathrm{THz}$ radiation have been developed including surface emitters [1-4], contactless $p-i-n$ emitters $[5,6]$, and photoconductive (PC) emitters [7-10]. The PC emitters are considered as the most efficient $\mathrm{THz}$ sources. In the present work, the results of the semiclassical Monte Carlo simulations of the transient dynamics of the photoexcited electron-hole plasma in GaAs- based $n$ - and $p$-doped surface emitters, contactless GaAs $p-i-n$ emitters, and GaAs PC emitters are presented. The compositions of the surface and $p-i-n$ emitters are optimized for maximum efficiency. The efficiencies of the optimized surface and $p-i-n$ emitters are compared with the efficiency of the PC emitter.

\section{Emitter models}

The model of the GaAs band structure as well as the phonon and impurity scattering processes are considered following Ref. [11]. The multi-channel intercarrier scattering, the Pauli exclusion principle, and the photoexcitation of electron-hole pairs are taken into account. The central photon energy is taken as $1.55 \mathrm{eV}$. The full width at half maximum of the temporal light intensity is set to $100 \mathrm{fs}$. The exponential spatial profile of the density of photoexcited carriers is

*corresponding author; e-mail: reklaitis@pfi.lt 
considered for the surface and $p-i-n$ emitters. The simulations are performed in the one-dimensional real space and in the three-dimensional momentum space. The simulations of the surface emitters and contactless $p-i-n$ emitter are started from the equilibrium carrier ensemble [12]. The simulations of the photoexcitation in the PC emitter are started after the nonequilibrium steady-state electron and potential distributions are established.

For $n$ - and $p$-doped surface emitters, the profile of doping concentration is taken as uniform. The thickness of the simulated structures is $4 \mu \mathrm{m}$, which essentially exceeds the width of depletion zone at the lowest doping concentration of $5 \times 10^{14} \mathrm{~cm}^{-3}$ considered in this work. The structure of the contactless $p-i-n$ emitters is the same as the structure studied in [11] except for the thickness of the intrinsic $i$-layer, which is varied in the present work. The PC emitter is considered as a one-dimensional lateral $n^{+}-i-n^{+}$diode biased to a fixed voltage. The electrodes of the PC emitter are treated as doped to $10^{20} \mathrm{~cm}^{-3} n^{+}$contacts. The electrons are injected from the contacts with the equilibrium Fermi-Dirac distribution [12]. The photoexcitation of electron-hole pairs is taken as spatially uniform over the whole volume of the PC emitter. In order to consider the depth-dependent exponential decay of carrier concentration in a one-dimensional model of the PC emitter, the depth-dependent density of photoexcited carriers is replaced by the carrier density averaged over the excitation depth.

The energy and spectrum of $\mathrm{THz}$ radiation are obtained from the simulated transient current of photoexcited carriers. The time-dependent $\mathrm{THz}$ power $P(t)$ radiated in all directions by the electric dipole moment $\boldsymbol{D}(t)$ and the first derivative of the dipole moment are evaluated as [13]:

$$
P(t)=\frac{1}{6 \pi \varepsilon c^{3}}\left(\frac{\mathrm{d}^{2} \boldsymbol{D}(t)}{\mathrm{d} t^{2}}\right)^{2} \text { and } \frac{\mathrm{d} \boldsymbol{D}(t)}{\mathrm{d} t}=\int \boldsymbol{j}(\boldsymbol{r}, t) \mathrm{d} V,
$$

where $\varepsilon$ is the vacuum permittivity, $c$ is the speed of light, $\boldsymbol{j}(\boldsymbol{r}, t)$ is the current density of electrons and holes, and the integration is taken over the volume $V$ of the photoexcitation. The total $\mathrm{THz}$ energy radiated in all directions is obtained by the integration of $P(t)$ over the duration of transient evolution of photoexcited carriers. The spectrum of radiation is found from the Fourier analysis of $P(t)$. The calculations are carried out for the same illumination area $S=10^{4} \mu \mathrm{m}^{2}$ for all three types of the emitters. For the PC emitter, the distance between electrodes is taken as $5 \mu \mathrm{m}[9,10]$, and the length of electrodes is set to $2 \mathrm{~mm}$.

\section{Results}

In Fig. 1, the transient currents of the electron-hole plasma excited in $n$ - and $p$-GaAs surface emitters are presented. As the doping concentration $N$ increases, the surface field strength rises as $N^{1 / 2}[1]$, and the amplitude of transient current increases. However, along with this, the width of depletion zone reduces as $N^{-1 / 2}$ [1], and the amplitude of the transient current tends to decrease due to the reduced part of excited carriers contributing to the transient current. The 
maximum efficiency is determined by the interplay between the surface electric field strength and the number of carriers contributing to the transient current. The transient current is also reduced by the electron intervalley scattering into the upper $L$ and $X$ valleys. The intervalley scattering is important at doping concentrations above $10^{16} \mathrm{~cm}^{-3}$. The results of simulations show that the optimal doping concentration for $n$ - and $p$-GaAs surface emitters is close to $10^{16} \mathrm{~cm}^{-3}$ and to $5 \times 10^{15} \mathrm{~cm}^{-3}$, respectively (see Fig. 2).
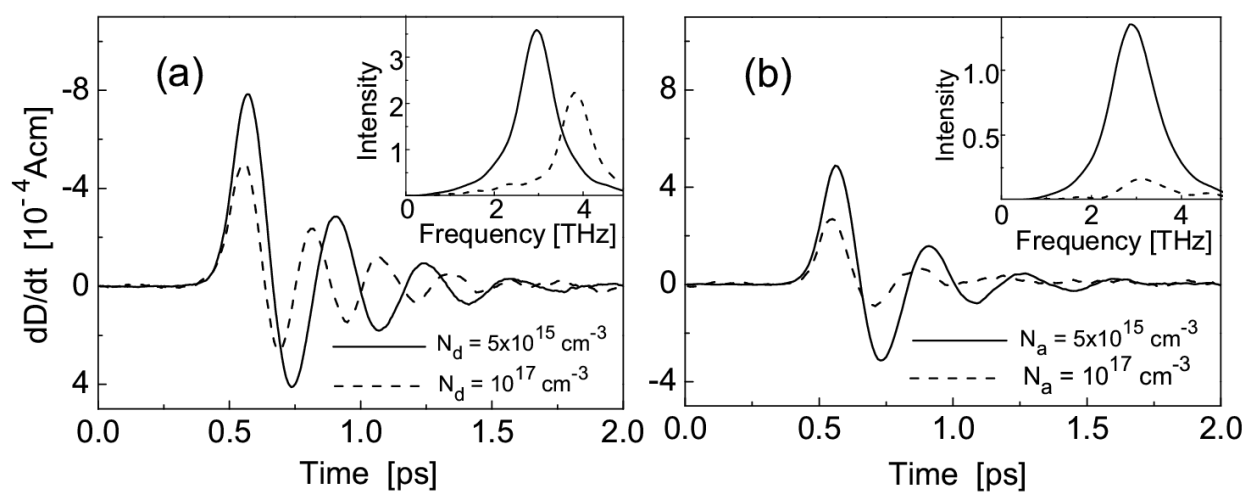

Fig. 1. Wave forms of the dipole moment derivative after optical pulse injection in surface emitters doped to $5 \times 10^{15} \mathrm{~cm}^{-3}$ (solid curves) and to $10^{17} \mathrm{~cm}^{-3}$ (dashed curves). (a): $n$-GaAs; (b): $p$-GaAs. The peak of the optical pulse is at $t=0.5$ ps. The insets show the corresponding Fourier spectra of radiated intensity. The excitation density is $10^{17} \mathrm{~cm}^{-3}$.

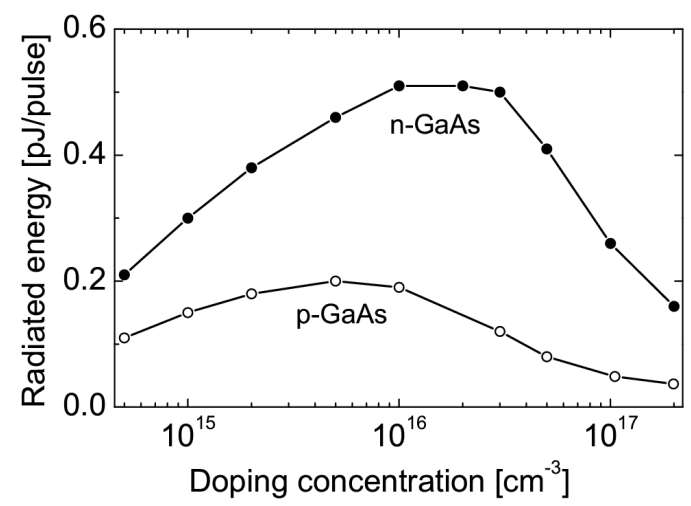

Fig. 2. THz energy radiated from $n$-doped (filled circles) and $p$-doped (open circles) GaAs surface emitters vs. doping concentration. The excitation density is $10^{17} \mathrm{~cm}^{-3}$.

A similar situation takes place in the contactless $p-i-n$ emitter. The transient response of the first derivative of the dipole moment in the $p-i-n$ emitter 
is presented in Fig. 3. The efficiency of $p-i-n$ emitters is determined by the interplay between the built-in electric field strength $E_{i}$ and the number of carriers contributing to the transient current. The value of $E_{i}$ in the $p-i-n$ structure can be estimated as $E_{i}=\varepsilon_{\mathrm{g}} / e L_{i}$, where $\varepsilon_{\mathrm{g}}$ is the band-gap energy, $e$ is electron charge, and $L_{i}$ is the thickness of $i$-layer. The built-in electric field increases as $L_{i}$ becomes reduced. However, along with this, the number of carriers, which make a contribution to the transient current is reduced. The maximum power is achieved when the value of $L_{i}$ is close to $0.4 \mu \mathrm{m}$ (see Fig. 4).

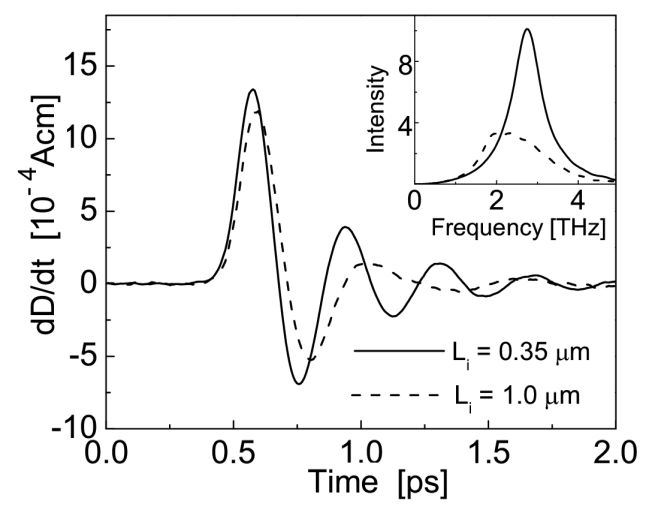

Fig. 3. Wave forms of the dipole moment derivative after optical pulse in $p-i-n$ emitter with $i$-layer thickness of $0.35 \mu \mathrm{m}$ (solid curve) and of $1 \mu \mathrm{m}$ (dashed curve). The peak of the optical pulse is at $t=0.5$ ps. The inset shows the corresponding Fourier spectra of radiated intensity. The excitation density is $10^{17} \mathrm{~cm}^{-3}$.

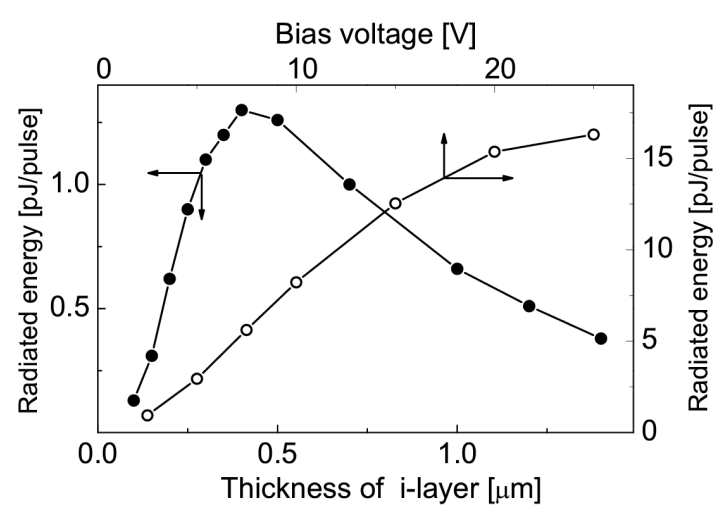

Fig. 4. THz energy radiated from $p-i-n$ structure vs. thickness of $i$-layer (filled circles) and from PC emitter vs. bias voltage (open circles). The excitation density is $10^{17} \mathrm{~cm}^{-3}$.

The results of the simulations of the carrier response in the $\mathrm{PC}$ emitter are presented in Fig. 5. The spectra of radiated intensity are in good agreement 
with the experimental data $[9,10]$. The simulations show that during the time interval of 1 ps after excitation, the transient current is primarily determined by the overshoot of the electron drift velocity. The subsequent slow decay in the photocurrent is controlled by the extraction of photoexcited carriers from the $i$ layer. The applied external electric field is screened by the spatial separation of electrons and holes resulting in the reduced rate of extraction of photoexcited carriers from the $i$-layer.

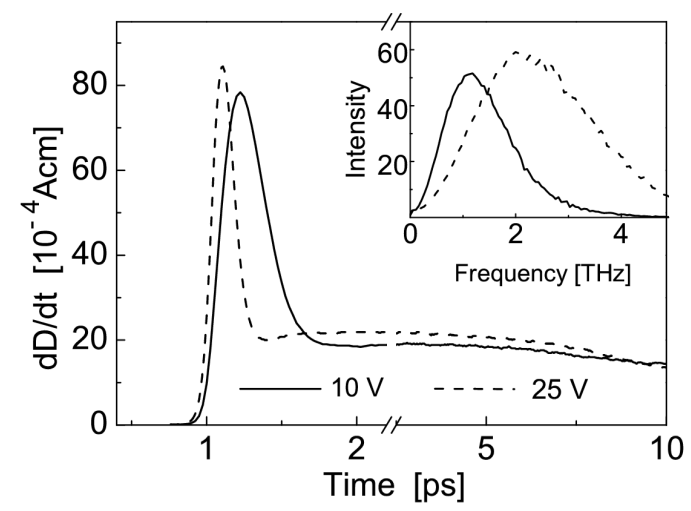

Fig. 5. Wave forms of the dipole moment derivative after optical pulse in photoconductive $\mathrm{THz}$ emitter biased to $10 \mathrm{~V}$ (solid curve) and to $25 \mathrm{~V}$ (dashed curve). The peak of the optical pulse is at $t=1 \mathrm{ps}$. The inset shows the corresponding Fourier spectra of radiated intensity. The excitation density is $10^{17} \mathrm{~cm}^{-3}$.

The comparison of $\mathrm{THz}$ energies radiated by the $n$ - and $p$-GaAs surface emitters, by the contactless $p-i-n$ emitter, and by the PC emitter shows that the energy radiated by the $\mathrm{PC}$ emitter exceeds the energy radiated by the rest of the emitters by more than one order of magnitude. The obtained results also show that the efficiency of the contactless $p-i-n$ emitter exceeds the efficiency of $n$-doped and $p$-doped surface emitters. We note that the dipole moment in the surface and $p-i-n$ emitters are oriented perpendicularly to the surface. Therefore, only a small fraction of the generated $\mathrm{THz}$ energy is extracted from the surface and from the $p-i-n$ emitters.

In summary, Monte Carlo simulation of GaAs-based pulsed THz emitters was carried out. The simulation shows that the efficiency of surface emitters depends on the doping concentration. The efficiency of the contactless $p-i-n$ emitters depends on the thickness of the $i$-layer. The optimal doping concentrations for the surface GaAs emitters and the optimal length of the $i$-layer for the contactless $p-i-n$ emitter are estimated. It was obtained that the $\mathrm{THz}$ energy radiated by the photoconductive emitter essentially exceeds the $\mathrm{THz}$ energy radiated by the surface and $p-i-n$ emitters. 


\section{Acknowledgments}

The work was supported by Lithuanian State Science and Studies Foundation under contract C-07004.

\section{References}

[1] T. Dekorsy, T. Pfeifer, W. Kütt, H. Kurz, Phys. Rev. B 47, 3842 (1993).

[2] R. Kersting, J.N. Heyman, G. Strasser, K. Unterrainer, Phys. Rev. B 58, 4553 (1998).

[3] J.N. Heyman, P. Neocleous, D. Hebert, P.A. Crowell, T. Müller, K. Unterrainer, Phys. Rev. B 64, 085202 (2001).

[4] J.S. Hwang, H.C. Lin, K.I. Lin, X. C. Zhang, Appl. Phys. Lett. 87, 121107 (2005).

[5] W. Sha, A.L. Smirl, W.F. Tseng, Phys. Rev. Lett. 74, 4273 (1995).

[6] R. Kersting, K. Unterrainer, G. Strasser, H.F. Kauffmann, E. Gornik, Phys. Rev. Lett. 79, 3038 (1997).

[7] J.T. Darrow, X.-C. Zhang, D.H. Auston, Appl. Phys. Lett. 58, 25 (1991).

[8] G. Zhao, R.N. Schouten, N. van der Valk, W.Th. Wenckebach, P.C.M. Planken, Rev. Sci. Instrum. 73, 1715 (2002).

[9] T.A. Liu, M. Tani, C.L. Pan, J. Appl. Phys. 93, 2996 (2003).

[10] A. Dreyhaupt, S. Winnerl, T. Dekorsy, M. Helm, Appl. Phys. Lett. 86, 121114 (2005).

[11] A. Reklaitis, Phys. Rev. B 74, 165305 (2006).

[12] A. Reklaitis, Appl. Phys. Lett. 86, 262110 (2005).

[13] G.S. Smith, An Introduction to Classical Electromagnetic Radiation, Cambridge University Press, Cambridge 1997, 459. 